Pacific

Journal of

Mathematics

\title{
CELLULARITY OF CERTAIN QUANTUM ENDOMORPHISM ALGEBRAS
}

HeNNing H. ANDERSEn, Gustav I. LeHRER AND Ruibin Zhang 


\title{
CELLULARITY OF CERTAIN QUANTUM ENDOMORPHISM ALGEBRAS
}

\author{
HenNing H. ANDERsen, Gustav I. LeHRER AND Ruibin Zhang
}

Dedicated to the memory of Robert Steinberg.

For any ring $\tilde{A}$ such that $\mathbb{Z}\left[q^{ \pm 1 / 2}\right] \subseteq \tilde{A} \subseteq \mathbb{Q}\left(q^{1 / 2}\right)$, let $\Delta_{\tilde{A}}(d)$ be an $\tilde{A}$-form of the Weyl module of highest weight $d \in \mathbb{N}$ of the quantised enveloping algebra $\mathrm{U}_{\tilde{A}}$ of $\mathfrak{s l}_{2}$. For suitable $\tilde{A}$, we exhibit for all positive integers $r$ an explicit cellular structure for $\operatorname{End}_{\mathrm{U}_{\tilde{A}}}\left(\Delta_{\tilde{A}}(d)^{\otimes r}\right)$. This algebra and its cellular structure are described in terms of certain Temperley-Lieb-like diagrams. We also prove general results that relate endomorphism algebras of specialisations to specialisations of the endomorphism algebras. When $\zeta$ is a root of unity of order bigger than $\boldsymbol{d}$ we consider the $U_{\zeta}$-module structure of the specialisation $\Delta_{\zeta}(d)^{\otimes r}$ at $q \mapsto \zeta$ of $\Delta_{\tilde{A}}(d)^{\otimes r}$. As an application of these results, we prove that knowledge of the dimensions of the simple modules of the specialised cellular algebra above is equivalent to knowledge of the weight multiplicities of the tilting modules for $U_{\zeta}\left(\mathfrak{s l}_{2}\right)$. As an example, in the final section we independently recover the weight multiplicities of indecomposable tilting modules for $\mathbf{U}_{\zeta}\left(\mathfrak{s l}_{2}\right)$ from the decomposition numbers of the endomorphism algebras, which are known through cellular theory.

\section{Introduction}

1A. Notation. Let $A$ be the ring $\mathbb{Z}\left[q^{ \pm 1 / 2}\right]$ where $q$ is an indeterminate, and let $\mathrm{U}_{A}$ be the Lusztig $A$-form $[1988 ; 1990 ; 1993]$ of the quantised enveloping algebra $\mathrm{U}_{q}\left(\mathfrak{s l}_{2}\right)$ [Drinfeld 1987; Jimbo 1986; Chari and Pressley 1994], which has basis consisting of products of "divided powers" of the generators of $\mathfrak{s l}_{2}$ and binomials in the Cartan generators. Let $\Delta_{A}(d)$ be the Weyl module for $\mathrm{U}_{A}$ with highest weight $d \in \mathbb{N}$. This has dimension $d+1$ and quantum dimension equal to the quantum number $[d+1]$, where for any integer $n$,

$$
[n]=[n]_{q}:=\frac{q^{n}-q^{-n}}{q-q^{-1}} .
$$

MSC2010: primary 17B37, 20G42; secondary 81R50.

Keywords: quantum invariants, cellular algebras, tilting modules. 
For any commutative $A$-algebra $\tilde{A}$, we write $\mathrm{U}_{\tilde{A}}:=\tilde{A} \otimes_{A} \mathrm{U}_{A}$, and similarly for $\Delta_{\tilde{A}}(d)$, etc. For any positive integer $r$, let $E_{r}(d, \tilde{A}):=\operatorname{End}_{\mathrm{U}_{\tilde{A}}}\left(\Delta_{\tilde{A}}(d)^{\otimes r}\right)$.

Let $s_{1}, \ldots, s_{N-1}$ be the standard Coxeter generators of $\operatorname{Sym}_{N}$. For $w \in \operatorname{Sym}_{N}$, write $\ell(w)$ for its length as a word in the generators $s_{i}$, and define the left set $L(w):=\left\{i \mid \ell\left(s_{i} w\right)<\ell(w)\right\}$; the right set $R(w)$ is defined similarly.

1B. The main result. Let $K=\mathbb{Q}\left(q^{1 / 2}\right)$ be the field of fractions of $A$. Writing $B_{r}$ for the $r$-string braid group ( $r$ a positive integer), it is known that there is an action of $B_{r}$ on $\Delta_{A}(d)^{\otimes r}$, in which the standard generators of the braid group act on successive tensor factors via the $R$-matrix $\check{R}$. This is evident over $K$, and from [Lehrer and Zhang 2006; 2010] and [Andersen et al. 2008] or [Andersen 2012] (using [Kirillov and Reshetikhin 1990]) in the above integral form. This action respects the $\mathrm{U}_{\tilde{A}}$-action on the tensor space, and so there is a homomorphism

$$
\eta: \tilde{A} B_{r} \longrightarrow \operatorname{End}_{\mathrm{U}_{\tilde{A}}}\left(\Delta_{\tilde{A}}(d)^{\otimes r}\right)=E_{r}(d, \tilde{A}) .
$$

We define $A$ using $q^{1 / 2}$ instead of $q$ because then, with the usual definitions of $\mathrm{U}_{q}$, the $R$-matrix is defined over $A$ with respect to a basis of weight vectors.

In [Lehrer and Zhang 2006] it was shown that when $\tilde{A}=K, \eta$ is surjective. This provides a means of studying the relevant endomorphism algebras. When $d=2$ this surjectivity was proved in [Andersen 2012] for most $\tilde{A}$. We haven't been able to establish this result for $d>2$. However, inspired in part by the methods used in [loc. cit.] we show in this paper that the endomorphism algebras have a nice cellular structure, even though the $R$-matrix generators satisfy a polynomial equation of degree $d+1$.

We shall work with the Temperley-Lieb algebra $\operatorname{TL}_{N}(\tilde{A})$, which has generators $f_{i}, i=1, \ldots, N-1$ and relations

$$
\begin{cases}f_{i} f_{j} f_{i}=f_{i} & \text { if }|i-j|=1, \\ f_{i} f_{j}=f_{j} f_{i} & \text { if }|i-j|>1, \\ f_{i}^{2}=\left(q+q^{-1}\right) f_{i} . & \end{cases}
$$

This has an $\tilde{A}$-basis consisting of planar diagrams, as explained in [Graham and Lehrer 1996, §1] (see also [2003; 2004]); these are in one-to-one correspondence with the set of fully commutative elements of $\mathrm{Sym}_{N}$; see [Fan and Green 1997].

Theorem 1.1. Let $d \geq 1$ be an integer. For any $\tilde{A}$ such that $[d]$ ! is invertible in $\tilde{A}$, the algebra $E_{r}(d, \tilde{A})$ is isomorphic to a cellular subalgebra of $\mathrm{TL}_{r d}(\tilde{A})$. In particular, it has an $\tilde{A}$-basis labelled by planar diagrams $D \in \mathrm{TL}_{r d}(\tilde{A})$ such that $L(D), R(D) \subseteq\{d, 2 d, \ldots,(r-1) d\}$, where the left and right sets $L(D)$ and $R(D)$ are as in Definition 3.2 below.

We remark that the cellular subalgebra in Theorem 1.1 has an identity different from that of $\mathrm{TL}_{r d}(\tilde{A})$, and is therefore not a unital subalgebra. 
Note that the planar diagrams are labelled by the set $\operatorname{Sym}_{r d}^{c}$ of fully commutative elements in $\mathrm{Sym}_{r d}$; the requirement in the theorem is equivalent to taking those $w \in \operatorname{Sym}_{r d}^{c}$ such that $L(w), R(w) \subseteq\{d, 2 d, \ldots,(r-1) d\}$ (see [Fan and Green 1997]).

We shall give further details of the cellular structure below, both in terms of diagrams, and in terms of pairs of standard tableaux.

\section{The case $d=1$}

2A. The Temperley-Lieb action. It is known (see, for example, [Lehrer and Zhang $2010, \S 3.4])$ that in this case, the $R$-matrix acts on $\Delta_{K}(1)^{\otimes 2}$ with eigenvalues $q^{1 / 2}$ and $-q^{3 / 2}$. If we adjust the map $\eta$ of (1-1) by sending the generators to $T_{i}:=q^{1 / 2} R_{i}$, where $R_{i}$ is the relevant $R$-matrix, then $\eta$ factors through the algebra $H_{r}(A):=A B_{r} /\left\langle\left(T_{i}+q^{-1}\right)\left(T_{i}-q\right)\right\rangle$, which is well known to be the Hecke algebra, and has $A$-basis $\left\{T_{w} \mid w \in \operatorname{Sym}_{r}\right\}$. We therefore have, after tensoring with $\tilde{A}$,

$$
\mu: H_{r}(\tilde{A}) \longrightarrow \operatorname{End}_{\mathrm{U}_{\tilde{A}}}\left(\Delta_{\tilde{A}}(1)^{\otimes r}\right)=E_{r}(1, \tilde{A}) .
$$

Moreover it is a special case of the main result of [Du et al. 1998] (see also [Andersen et al. 2008]) that $\mu$ is surjective for any choice of $\tilde{A}$, even when $\tilde{A}$ is taken to be $A$. Further, the arguments in [Lehrer and Zhang 2010, Theorem 3.5], generalised to the integral case, show that the kernel of $\mu$ is the ideal generated by the element $a_{3}:=\sum_{w \in \operatorname{Sym}_{3}}(-q)^{-\ell(w)} T_{w}$; hence, for any $\tilde{A}$, we have an isomorphism

$$
\eta: H_{r}(\tilde{A}) /\left\langle a_{3}\right\rangle \cong \mathrm{TL}_{r}(\tilde{A}) \stackrel{\sim}{\longrightarrow} \operatorname{End}_{\mathrm{U}_{\tilde{A}}}\left(\Delta_{\tilde{A}}(1)^{\otimes r}\right)=E_{r}(1, \tilde{A}),
$$

where $\operatorname{TL}_{r}(\tilde{A}):=H_{r}(\tilde{A}) /\left\langle a_{3}\right\rangle$ is the $r$-string Temperley-Lieb algebra. The generator $f_{i}$ acts as $q-T_{i}$ on $\Delta_{\tilde{A}}(1)^{\otimes r}$. It is easily shown that $f_{i}^{2}=\left(q+q^{-1}\right) f_{i}$, and that the other Temperley-Lieb relations are satisfied.

2B. Projection to $\Delta_{\tilde{A}}(d)$. Now it is elementary that

$$
\Delta_{K}(1)^{\otimes d} \cong \Delta_{K}(d) \oplus \Delta^{\prime},
$$

where $\Delta^{\prime}$ is the direct sum of simple modules $\Delta_{K}(i)$ with $i<d$. We therefore have a canonical projection $p_{d}: \Delta_{K}(1)^{\otimes d} \longrightarrow \Delta_{K}(d)$, which may be considered an element of $E_{d}(1, K)=\operatorname{End}_{\mathrm{U}_{K}}\left(\Delta_{K}(1)^{\otimes d}\right)$.

Lemma 2.1. The projection $p_{d}$ is the image under $\mu$ (see (2-1)) of the element $e_{d}:=P_{d}(q)^{-1} \sum_{w \in \operatorname{Sym}_{d}} q^{\ell(w)} T_{w} \in H_{d}(\tilde{A})$, where $P_{d}(q)=q^{d(d-1) / 2}[d] !$.

Proof. We begin by showing that for $i=1, \ldots, d-1$,

$$
T_{i} p_{d}=p_{d} T_{i}=q p_{d}
$$

as endomorphisms of $\Delta_{K}(1)^{\otimes d}$. 
By symmetry, it suffices to prove (2-4) for $i=1$. Now

$$
\begin{aligned}
\Delta_{K}(1)^{\otimes d} & =\Delta_{K}(1) \otimes \Delta_{K}(1) \otimes \Delta_{K}(1)^{\otimes(d-2)} \\
& \cong\left(\Delta_{K}(0) \oplus \Delta_{K}(2)\right) \otimes \Delta_{K}(1)^{\otimes(d-2)} \\
& \cong\left(\Delta_{K}(0) \otimes \Delta_{K}(1)^{\otimes(d-2)}\right) \oplus\left(\Delta_{K}(2) \otimes \Delta_{K}(1)^{\otimes(d-2)}\right)
\end{aligned}
$$

But $p_{d}$ acts as zero on the first summand (since the highest occurring weight is $d-2$ ) and $T_{1}$ acts as $q$ on the second summand. This proves the relation (2-4). Now since $f_{i}=\mu\left(q-T_{i}\right)$, this shows that $p_{d}$ is the "Jones idempotent" of $\operatorname{TL}_{d}(K)$, defined by the relations $f_{i} p_{d}=p_{d} f_{i}=0$ for all $i$.

It follows that if $p_{d}^{\prime}$ is the unique idempotent in $H_{d}(K)$ corresponding to the algebra homomorphism $T_{w} \mapsto q^{\ell(w)}$, then $p_{d}=\mu\left(p_{d}^{\prime}\right)$. But this idempotent is precisely the element $e_{d}$ in the statement.

The next statement is immediate.

Corollary 2.2. Let $\tilde{A}=A\left[[d] !^{-1}\right]$. Then

$$
\Delta_{\tilde{A}}(1)^{\otimes r d} \cong \Delta_{\tilde{A}}(d)^{\otimes r} \oplus \Gamma,
$$

where $\Gamma$ is a $\mathrm{U}_{\tilde{A}}$-submodule, and the corresponding projection $p \in \operatorname{End}_{r d}(1, \tilde{A})$ such that $p\left(\Delta_{\tilde{A}}(1)^{\otimes r d}\right)=\Delta_{\tilde{A}}(d)^{\otimes r}$ is given by $p=p_{d}^{\otimes r}$, where we now consider $p_{d}$ as an element of $E_{d}(1, \tilde{A}) \subset E_{d}(1, K)$.

\section{Endomorphisms of $\Delta_{\tilde{A}}(d)^{\otimes r}$}

3A. Identification of $\boldsymbol{E}_{\boldsymbol{r}}(\boldsymbol{d}, \tilde{\boldsymbol{A}})$. Throughout this section we take $\tilde{A}$ to be $\tilde{A}=$ $A\left[[d] !^{-1}\right]$. Recall that $E_{r}(d, \tilde{A})=\operatorname{End}_{\mathrm{U}_{\tilde{A}}}\left(\Delta_{\tilde{A}}(d)^{\otimes r}\right)$. We are now in a position to identify $E_{r}(d, \tilde{A})$ on the nose, as a subalgebra of $\operatorname{TL}_{r d}(\tilde{A}) \cong \operatorname{End}_{\mathrm{U}_{\tilde{A}}}\left(\Delta_{\tilde{A}}(1)^{\otimes r d}\right)$. This will lead to the identification of the cellular structure on $E_{r}(d, \tilde{A})$.

Proposition 3.1. There is an isomorphism $E_{r}(d, \tilde{A}) \stackrel{\sim}{\longrightarrow} p \mathrm{TL}_{r d}(\tilde{A}) p$, where $p$ is the idempotent $p=p_{d}^{\otimes r}$ of $\mathrm{TL}_{r d}(\tilde{A})$ described above.

Proof. For any endomorphism $\alpha \in E_{r}(d, \tilde{A})$ we obtain an endomorphism $\tilde{\alpha}$ of $\Delta_{\tilde{A}}(1)^{\otimes r d}$ by extending $\alpha$ by zero, using the decomposition (2-5), that is, by defining $\tilde{\alpha}$ to be zero on $\Gamma$. The map $\alpha \mapsto \tilde{\alpha}$ is an inclusion $E_{r}(d, \tilde{A}) \hookrightarrow E_{r d}(1, \tilde{A})$, and its image is clearly the space of endomorphisms $\beta \in E_{r d}(1, \tilde{A})$ such that $\operatorname{ker}(\beta) \supseteq \Gamma$ and $\operatorname{Im}(\beta) \subset \Delta_{\tilde{A}}(d)^{\otimes r}$ (as in the decomposition (2-5)). This image is $p \operatorname{TL}_{r d}(\tilde{A}) p$.

3B. Temperley-Lieb diagrams. The key step in proving cellularity is the identification of a certain $\tilde{A}$-basis of $p \mathrm{TL}_{r d}(\tilde{A}) p$. This will be done in terms of certain diagrams. The Temperley-Lieb algebra $\operatorname{TL}_{r d}(\tilde{A})$ has $\tilde{A}$-basis consisting of planar diagrams from $r d$ to $r d$, in the language of [Graham and Lehrer 1998]. These 


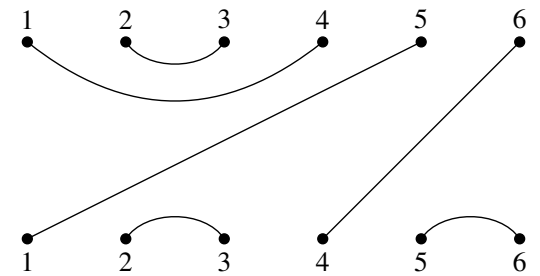

Figure 1. A planar diagram from 6 to 6 .

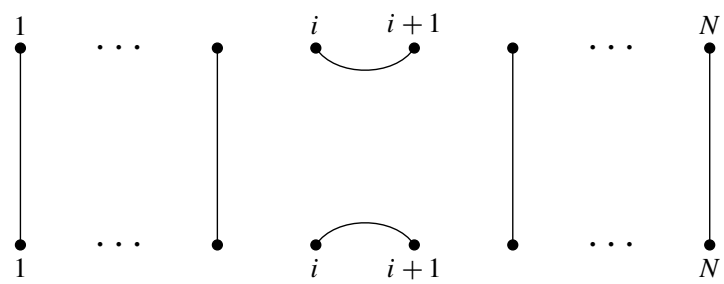

Figure 2. The generator $f_{i}$ as a planar diagram from $N$ to $N$.

diagrams are in bijection with the set $\operatorname{Sym}_{r d}^{c}$ of fully commutative elements [Fan and Green 1997] of $\mathrm{Sym}_{r d}$, which in turn is in bijection with those elements of Sym $_{r d}$ which correspond, under the Robinson-Schensted correspondence, to pairs of standard tableaux with two rows.

We shall describe now how to obtain a pair $(S(D), R(D))$ of standard tableaux directly from a planar diagram $D$. We use the planar diagram from 6 to 6 in Figure 1 to illustrate the description.

Each planar diagram from $N$ to $N$ consists of a set of $N$ nonintersecting $\operatorname{arcs}$. These may be through-arcs, joining an upper node to a lower node, or upper (top to top) or lower (bottom to bottom). The latter two are referred to as horizontal arcs. The diagrams are multiplied in the usual way, by concatenation, with each closed circle being replaced by [2] $=q+q^{-1}$. The generator $f_{i}$ corresponds to the diagram in Figure 2. Note that if there are $t$ through-arcs, then there are equally many top arcs and bottom arcs, and if this number is $k$, then $t+2 k=N$.

Now to each such planar diagram $D$, we associate an ordered pair $(S(D), T(D))$ of standard tableaux with two rows, as follows. Let $i_{1}, \ldots, i_{k}$ be the right nodes of the upper arcs written in ascending order. Then $S(D)$ has second row $i_{1}, \ldots, i_{k}$, and first row the complement of $\left\{i_{1}, \ldots, i_{k}\right\}$, written in ascending order. Note that the first row has $t+k \geq k$ elements. The tableau $T(D)$ is defined similarly, using the sequence $j_{1}, \ldots, j_{k}$ of right ends of the lower arcs. Note that both $S(D)$ and $T(D)$ correspond to the partition $(t+k, k)$, and hence the diagram corresponds via 
the Robinson-Schensted correspondence to an element $w(D) \in \operatorname{Sym}_{N}$, which is fully commutative (see [Fan and Green 1997, Definition 3.3.1]).

Say that a horizontal arc is small if its vertices are $i, i+1$ for some $i$.

Definition 3.2. The left set $L(D)$ of a planar diagram $D$ is the set of left vertices of the small upper arcs of $D$. Similarly, the right set $R(D)$ is the set of left vertices of the small lower arcs of $D$.

It is well known, and proved in a straightforward way using the RobinsonSchensted correspondence, that in the notation from Section 1A we have $L(D)=$ $L(w(D))$, and similarly $R(D)=R(w(D))$.

For the diagram $D$ in Figure $1, L(D)=\{2\}$, while $R(D)=\{2,5\}$. The tableaux $S(D)$ and $T(D)$ are given by

$$
S(D)=\begin{array}{|l|l|l|l}
\hline 1 & 2 & 5 & 6 \\
\hline 3 & 4 &
\end{array} \quad \text { and } T(D)=\begin{array}{|l|l|l|l|}
\hline 1 & 2 & 4 & 5 \\
\hline 3 & 6 & &
\end{array} .
$$

Note that if $\mathscr{D}(S):=\{i \mid i+1$ is in a lower row than $i\}$ is the descent set of a standard tableau $S$, then $L(D)=\mathscr{D}(S(D))$ and $R(D)=\mathscr{D}(T(D))$.

\section{Proof of the main theorem}

In this section we prove Theorem 1.1, and give some of its consequences. We keep the convention $\tilde{A}=A\left[([d] !)^{-1}\right]$ from Section 3 .

4A. A key lemma. We begin by proving the following key result.

Lemma 4.1. The Â-algebra $p \mathrm{TL}_{d r}(\tilde{A}) p$ has $\tilde{A}$-basis given by the set of elements $p D p$, where $D$ is a diagram in $\mathrm{TL}_{d r}(\tilde{A})$ such that

$$
L(D) \cup R(D) \subseteq\{d, 2 d, \ldots,(r-1) d\} .
$$

Proof. The $\tilde{A}$-algebra $E_{r}(d, \tilde{A}) \cong p \mathrm{TL}_{r d}(\tilde{A}) p$ is evidently spanned by the elements $p D p$, where $D$ ranges over planar diagrams from $r d$ to $r d$. But for $i=1, \ldots, d-1$, we have seen that $p_{d} f_{i}=f_{i} p_{d}=0$. It follows that $p D p=0$ unless $L(D)$ and $R(D)$ are both contained in $\{d, 2 d, \ldots,(r-1) d\}$. Let $\mathscr{B}(d, r)$ be the set of planar diagrams satisfying these conditions. By the above remarks, it will suffice to show that

$$
\{p D p \mid D \in \mathscr{B}(d, r)\} \text { is linearly independent. }
$$

To prove (4-1) it suffices to work over the field $K$; in particular we are reduced to showing that

$$
|\dddot{B}(d, r)|=\operatorname{dim}_{K}\left(\operatorname{End}_{\mathrm{U}_{K}}\left(\Delta_{K}(d)^{\otimes r}\right) .\right.
$$


We shall prove (4-2) essentially by showing that both sides of (4-2) satisfy the same recurrence. Let us begin with the left side.

Observe that if a diagram $D \in \mathscr{B}(d, r)$ has $t$ through-arcs, it may be thought of as a pair of diagrams $D_{1}, D_{2}$, where the $D_{i}$ are monic diagrams from $t$ to $r d$. Recall that a diagram from $t$ to $N(t \leq N)$ is monic if it has $t$ through-arcs. One thinks of $D_{1}$ as the top half of $D$, and $D_{2}$ as the ${ }^{*}$ of the bottom half of $D$, where ${ }^{*}$ is the cellular involution on the Temperley-Lieb category that reflects diagrams in a horizontal line. It follows that if we write $|\mathscr{B}(d, r)|=b(d, r)$ and $|\mathscr{B}(d, r ; t)|=b(d, r ; t)$, where $\mathscr{B}(d, r ; t)$ is the set of monic planar diagrams $D: t \rightarrow r d$ such that $L(D) \subseteq\{d, 2 d, \ldots,(r-1) d\}$, then

$$
b(d, r)=\sum_{0 \leq t \leq d r} b(d, r ; t)^{2} .
$$

Now consider the right side of (4-2). Define the positive integers $m(d, r ; t)$ by

$$
\Delta_{K}(d)^{\otimes r} \cong \bigoplus_{t=0}^{d r} m(d, r ; t) \Delta_{K}(t) .
$$

Thus the $m(d, r ; t)$ are multiplicities, and $m(d, r ; t)=0$ unless $t \equiv r d(\bmod 2)$. Moreover, we obviously have, if $m(d, r):=\operatorname{dim}_{K}\left(\operatorname{End}_{\mathrm{U}_{K}}\left(\Delta_{K}(d)^{\otimes r}\right)\right.$,

$$
m(d, r)=\sum_{0 \leq t \leq d r} m(d, r ; t)^{2} .
$$

It is clear that in view of (4-3) and (4-5), the lemma will follow if we prove that for all $d, r$ and $t$,

$$
m(d, r ; t)=b(d, r ; t)
$$

We shall prove (4-6) by induction on $r$. If $r=1$, then

$$
m(d, 1 ; t)=b(d, 1 ; t)= \begin{cases}0 & \text { if } t \neq d \\ 1 & \text { if } t=d .\end{cases}
$$

Now by the Clebsch-Gordan formula, we have, for any integer $n$,

$$
\Delta_{K}(d) \otimes \Delta_{K}(n) \cong \Delta_{K}(d+n) \oplus \Delta_{K}(d+n-2) \oplus \cdots \oplus \Delta_{K}(|d-n|) .
$$

It follows that

$$
m(d, r+1 ; t)=\sum_{s=t-d}^{t+d} m(d, r ; s),
$$

where $m(d, r ; s)=0$ if $s<0$ or if $s>d r$.

We shall complete the proof of the lemma by showing that the numbers $b(d, r ; t)$ satisfy a recurrence analogous to (4-8). For this observe that any diagram $D$ in 


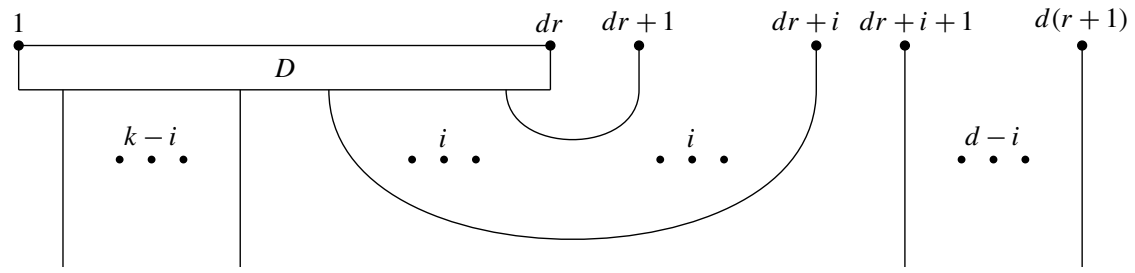

Figure 3. From diagram $D$ to diagram $D^{\prime}$.

$\mathscr{B}(d, r ; k)$ gives rise to a unique diagram in $\mathscr{B}(d, r+1 ; k+d-2 i)$, for $0 \leq i \leq$ $\min \{d, k\}$, as depicted in Figure 3, and each diagram $D^{\prime} \in \mathscr{B}(d, r+1 ; t)$ arises in this way from a unique diagram in $\mathscr{B}(d, r ; k)$ for a uniquely determined $k$. In fact, $k=t-d+2 i$ where $i$ is the number of arcs in $D^{\prime}$ whose right vertices belong to $\{d r+1, \cdots, d(r+1)\}$. It follows that

$$
b(d, r+1 ; t)=\sum_{s=t-d}^{t+d} b(d, r ; s),
$$

where $b(d, r ; s)=0$ if $s<0$ or if $s>d r$.

Comparing (4-8) with (4-9), and taking into account (4-7), it follows that $m(d, r ; k)=b(d, r ; k)$ for all $d, r$ and $k$. This completes the proof of (4-6) above, and hence of the lemma.

\section{B. Cellular structure.}

Proof of Theorem 1.1. We have seen that $E_{r}(d, \tilde{A}) \cong p \mathrm{TL}_{r d}(\tilde{A}) p$, and that the latter algebra has the basis $\mathscr{B}(d, r)$, as stated in the theorem. It remains only to show that $p \mathrm{TL}_{r d}(\tilde{A}) p$ has a cellular structure. Following [Graham and Lehrer 1996, Definition 1.1] we need to produce a cell datum $\left(\Lambda, M, C,{ }^{*}\right)$ for $p \operatorname{TL}_{r d}(\tilde{A}) p$.

Take $\Lambda$ to be the poset $\{t \in \mathbb{Z} \mid 0 \leq t \leq d r$ and $d r-t \in 2 \mathbb{Z}\}$, ordered as integers. For $t \in \Lambda$, let $M(t):=\mathscr{B}(d, r ; t)$, the set of monic planar diagrams $D: t \rightarrow d r$ such that $L(D) \subseteq\{d, 2 d, \ldots,(r-1) d\}$ (see Section 3B and the proof of Lemma 4.1). Then the map $C: \amalg_{t \in \Lambda} M(t) \times M(t) \longrightarrow p \mathrm{TL}_{r d}(\tilde{A}) p$ is defined by $C\left(D_{1}, D_{2}\right)=p D_{1} \circ D_{2}^{*} p$, where $\circ$ indicates concatenation of diagrams. We shall henceforth simply use juxtaposition to indicate composition in the Temperley-Lieb category. Since each diagram $D \in \mathscr{B}(r, d)$ is expressible uniquely as $D=D_{1} D_{2}^{*}$ for some $t \in \Lambda$ and $D_{1}, D_{2} \in M(t)$, it follows from Lemma 4.1 that $C$ is a bijection from $\amalg_{t \in \Lambda} M(t) \times M(t)$ to a basis of $p \mathrm{TL}_{r d}(\tilde{A}) p$. Finally, the anti-involution * is the restriction to $p \mathrm{TL}_{r d}(\tilde{A}) p$ of the anti-involution on $\mathrm{TL}_{d r}(\tilde{A})$, namely, reflection in a horizontal line. Since $p^{*}=p$, we have $C\left(D_{1}, D_{2}\right)^{*}=\left(p D_{1} D_{2}^{*} p\right)^{*}=p D_{2} D_{1}^{*} p=$ $C\left(D_{2}, D_{1}\right)$. 
If $S, T \in M(t)$, we shall write $C(S, T)=C_{S, T}^{t}$, and for this proof only, write

$$
\mathscr{A}=p \mathrm{TL}_{r d}(\tilde{A}) p \quad \text { and } \quad \mathscr{A}(<i)=\sum_{\substack{j<i, S, T \in M(j)}} \tilde{A} C_{S, T}^{j} .
$$

It remains only to prove the axiom (C3) of [Graham and Lehrer 1996, Definition 1.1]. For this, let $S_{1}, S_{2} \in M(s)$ and $T_{1}, T_{2} \in M(t)$. Then

$$
C_{S_{1}, S_{2}}^{s} C_{T_{1}, T_{2}}^{t}=p S_{1}\left(S_{2}^{*} p T_{1}\right) T_{2}^{*} p
$$

so that if $s<t$, the left side is in $\mathscr{A}(<t)$, and there is nothing to prove. Hence we take $s \geq t$.

Now $S_{2}^{*} p T_{1}$ is a morphism from $t$ to $s$, and hence is an $\tilde{A}$-linear combination of planar diagrams $D$ from $t$ to $s$. Thus the left side of (4-10) is an $\tilde{A}$-linear combination of elements of the form $p S_{1} D T_{2}^{*} p$. If $D$ is not monic, then $p S_{1} D T_{2}^{*} p \in \mathscr{A}(<t)$; if $D$ is monic, then clearly $p S_{1} D T_{2}^{*} p=p S^{\prime} T_{2}^{*} p$ for some monic $S^{\prime}: t \rightarrow d r$.

It follows from (4-10) that modulo $\mathscr{A}(<t), C_{S_{1}, S_{2}}^{s} C_{T_{1}, T_{2}}^{t}=\sum_{S \in \mathscr{B}(d, r ; t)} a(S) C_{S, T_{2}}^{t}$, and $a(S)$ is independent of $T_{2}$. This proves the axiom (C3), and hence the cellularity of $\mathscr{A}$. The proof of Theorem 1.1 is now complete.

\section{Endomorphism algebras and specialisation}

We shall prove in this section results showing how the multiplicities of the indecomposable summands of the specialisations of $\Delta_{A}(d)^{\otimes r}$ corresponding to homomorphisms $A \rightarrow k$ where $k$ is a field, relate to the dimensions of the simple modules for the corresponding endomorphism rings. It turns out that this is a consequence of a result on tilting modules which is valid for general quantum groups. Therefore in Sections 5A and 5B we deal with this general situation. Then in Section 5C we deduce the explicit consequences in our $\mathfrak{s l}_{2}$ case where we take advantage of our cellularity result from Section 4 on the endomorphism rings.

5A. Integral endomorphism algebras and specialisation. We now provide some rather general base change results for Hom-spaces between certain representations of quantum groups. So in this section we shall work with a general quantum group $\mathrm{U}_{q}$ over $K$ with integral form $\mathrm{U}_{A}$. We denote by $k$ an arbitrary field (in this section $k$ may even be any commutative noetherian $A$-algebra) made into an $A$-algebra by specializing $q$ to $\zeta \in k \backslash\{0\}$ and set $\mathrm{U}_{\zeta}=\mathrm{U}_{A} \otimes_{A} k$. When $M$ is a $\mathrm{U}_{A}$-module we write $M_{q}$ and $M_{\zeta}$ for the corresponding $\mathrm{U}_{q^{-}}$and $\mathrm{U}_{\zeta}$-modules, respectively.

For each dominant weight $\lambda$ we write $\Delta_{q}(\lambda), \Delta_{A}(\lambda)$ and $\Delta_{\zeta}(\lambda)$ for the Weyl modules for $\mathrm{U}_{q}, \mathrm{U}_{A}$ and $\mathrm{U}_{\zeta}$ respectively. Similarly, we have the dual Weyl modules $\nabla_{q}(\lambda), \nabla_{A}(\lambda)$ and $\nabla_{\zeta}(\lambda)$ respectively. Then it is well known that, writing $w_{0}$ for 
the longest element of the Weyl group,

$$
\nabla_{\zeta}(\lambda)=\Delta_{\zeta}\left(-w_{0} \lambda\right)^{*}
$$

and similarly for $\nabla_{A}(\lambda)$ and $\nabla_{q}(\lambda)$.

We shall make repeated use of the following result. For any two weights $\lambda, \mu \in X$, we have

$$
\operatorname{Ext}_{\mathrm{U}_{A}}^{i}\left(\Delta_{A}(\lambda), \nabla_{A}(\mu)\right)= \begin{cases}A & \text { if } \lambda=\mu \text { and } i=0 \\ 0 & \text { otherwise. }\end{cases}
$$

This is proved exactly as in the corresponding classical case (see, for example, [Jantzen 2003, Proposition II.B.4]) by invoking the quantised Kempf vanishing theorem proved in general in [Ryom-Hansen 2003].

Lemma 5.1. Let $M, N$ be $\mathrm{U}_{A}$-modules that are finitely generated as A-modules. If $M$ has a filtration by Weyl modules $\Delta_{A}(\lambda)$ and $N$ has a filtration by dual Weyl modules $\nabla_{A}(\mu)$, then $\operatorname{Hom}_{\mathrm{U}_{A}}(M, N)$ is a free A-module of rank equal to $\operatorname{dim}_{\mathbb{Q}(q)} \operatorname{Hom}_{\mathrm{U}_{q}}\left(M_{q}, N_{q}\right)$. Further, we have

$$
\operatorname{Hom}_{U_{\zeta}}\left(M_{\zeta}, N_{\zeta}\right) \simeq \operatorname{Hom}_{\mathrm{U}_{A}}\left(M_{A}, N_{A}\right) \otimes_{A} k
$$

Proof. We have a spectral sequence with $E_{2}$-terms

$$
E_{2}^{-p, q}=\operatorname{Tor}_{p}^{A}\left(\operatorname{Ext}_{\mathrm{U}_{A}}^{q}(M, N), k\right)
$$

converging to $\operatorname{Ext}_{\mathrm{U}_{\zeta}}^{q-p}\left(M_{\zeta}, N_{\zeta}\right)$. By (5-1) we have $E_{2}^{-p, q}=0$ if either $q>0$ or $q=0<p$. Hence the spectral sequence collapses and we can read off the result.

Corollary 5.2. Let $V$ be a $\mathrm{U}_{A}$-module which satisfies the assumption

$$
V^{*} \otimes_{A} V \text { has a } \nabla_{A} \text {-filtration. }
$$

Then $\operatorname{End}_{\mathrm{U}_{\zeta}}\left(V_{\zeta}^{\otimes r}\right) \simeq \operatorname{End}_{\mathrm{U}_{A}}\left(V^{\otimes r}\right) \otimes_{A} k$.

Proof. We have $\operatorname{End}_{\mathrm{U}_{A}}\left(V^{\otimes r}\right) \simeq \operatorname{Hom}_{\mathrm{U}_{A}}\left(\Delta_{A}(0),\left(V^{*} \otimes V\right)^{\otimes r}\right)$ because $\Delta_{A}(0)$ is the trivial $\mathrm{U}_{A}$-module $A$. By the assumption (5-2), we may apply Lemma 5.1 to obtain the statement.

As usual we denote by $\rho$ half the sum of the positive roots. Recall the concept of strongly multiplicity-free modules from [Lehrer and Zhang 2006]. A $\mathrm{U}_{q}$-module $V_{q}$ is strongly multiplicity-free if the weights of $\mathrm{U}_{q}$ occurring in $V_{q}$ form a chain in the usual ordering on weights.

There are significant cases where the above result applies:

Proposition 5.3. Suppose $V=\Delta_{A}(\lambda)$ for some dominant weight $\lambda$. Assume that $V_{q}$ is strongly multiplicity-free, and that $-w_{0} \lambda+\mu+\rho$ is dominant for each weight $\mu$ of $V$. Then $V^{*} \otimes V$ has a $\nabla_{A}$-filtration. 
Proof. Recall that $\mathrm{U}_{A}$ has a triangular decomposition $\mathrm{U}_{A}=\mathrm{U}_{A}^{+} \mathrm{U}_{A}^{0} \mathrm{U}_{A}^{-}$, and each weight $\mu$ defines a 1-dimensional representation of the subalgebra $\mathrm{U}_{A}^{0} \mathrm{U}_{A}^{-}$, which we also denote by $\mu$.

We have $V^{*}=\nabla_{A}\left(\lambda^{\prime}\right)$ where $\lambda^{\prime}=-w_{0} \lambda$. Moreover $\nabla_{A}$ is realised as the induction functor Ind $\mathrm{U}_{A}^{0} \mathrm{U}_{A}^{-}$. Hence by a standard property of induction,

$$
V^{*} \otimes V=\operatorname{Ind}_{\mathrm{U}_{A}^{0} \mathrm{U}_{A}^{-}}^{\mathrm{U}_{A}}\left(\lambda^{\prime}\right) \otimes V=\operatorname{Ind}_{\mathrm{U}_{A}^{0} \mathrm{U}_{A}^{-}}^{\mathrm{U}_{A}}\left(\lambda^{\prime} \otimes V\right),
$$

where in this formula the last occurrence of $V$ is its restriction to $\mathrm{U}_{A}^{0} \mathrm{U}_{A}^{-}$. Now the hypothesis that $V_{q}$ is strongly multiplicity-free implies that the weights of $V$ are linearly ordered. But the weights of $\lambda^{\prime} \otimes V$ are $\left\{\lambda^{\prime}+\mu\right\}$, where $\mu$ runs over the weights of $V$. This set is therefore a linearly ordered chain, and accordingly, $\lambda^{\prime} \otimes V$ has a $\mathrm{U}_{A}^{0} \mathrm{U}_{A}^{-}$-module filtration

$$
0=F_{0} \subset F_{1} \subset \cdots \subset F_{d}=\lambda^{\prime} \otimes V
$$

where $d=\operatorname{dim} V_{q}$, with the quotients $F_{i} / F_{i-1}$ running over the $\mathrm{U}_{A}^{0} \mathrm{U}_{A}^{-}$-modules $\lambda^{\prime}+\mu$. Our hypothesis, together with (the quantised) Kempf's vanishing theorem imply that the higher (degree $>0$ ) cohomology of the corresponding line bundles vanishes, and hence that induction is exact on this filtration. We therefore have a corresponding filtration of $\mathrm{U}_{A}$-modules

$$
0 \subset \nabla_{A}\left(F_{1}\right) \subset \cdots \subset \nabla_{A}\left(F_{d}\right)=\nabla_{A}\left(\lambda^{\prime} \otimes V\right)=V^{*} \otimes V .
$$

Corollary 5.4. The conclusion of Proposition 5.3 holds in the following cases.

(1) $V$ is a Weyl module with minuscule highest weight. This includes the natural modules in types $A, C$ and $D$ (but not type B).

(2) $V$ is any Weyl module for $\mathrm{U}_{A}\left(\mathfrak{s l}_{2}\right)$.

(3) $V$ is the Weyl module in type $G_{2}$ with highest weight $2 \alpha_{1}+\alpha_{2}$, where $\alpha_{1}$ and $\alpha_{2}$ denote the two simple roots, with $\alpha_{2}$ long.

Proof. When $V$ is minuscule, it is well known that for any weight $\mu$ of $V$ we have $\left(\mu, \alpha^{\vee}\right)= \pm 1$ or 0 , and hence (1) is clear. The case of $\mathfrak{s l}_{2}$ is evident, while in the case of type $G_{2}$, the weights of the Weyl module in question are the short roots, together with 0 . This easily gives (3).

5B. Multiplicities of tilting modules and dimensions of irreducibles. In this section we shall prove some rather general results which will allow us to relate multiplicities of indecomposable tilting summands in tensor powers of certain representations of quantum groups to the dimensions of simple modules for the corresponding endomorphism algebras. 
We note that the results of this section are similar in spirit to those of [Brundan and Kleshchev 1999, §3], which in turn have their genesis in some aspects of [Mathieu and Papadopoulos 1999, §3].

Theorem 5.5. Let $k$ be a field, $\mathrm{U}$ a $k$-algebra, and $M$ a finite-dimensional (over $k$ ) $\mathrm{U}$-module. Let $E=\operatorname{End}_{\mathrm{U}}(M)$, and assume that for each indecomposable direct summand $M^{\prime}$ of $M$, we have $E^{\prime} / \operatorname{Rad} E^{\prime} \simeq k$ where $E^{\prime}=\operatorname{End}_{\mathrm{U}}\left(M^{\prime}\right)$. Then

$$
\frac{E}{\operatorname{Rad} E} \simeq \bigoplus_{i} M_{d_{i}}(k)
$$

where $M_{d}(k)$ is the algebra of $n \times n$ matrices over $k, i$ runs over the isomorphism classes of indecomposable U-modules (of course only a finite number occur), and the $d_{i}$ are the multiplicities of the indecomposable summands of $M$.

Proof. Let $M=M_{1} \oplus M_{2} \oplus \cdots \oplus M_{n}$ be a decomposition of $M$ into indecomposables. Then any endomorphism $\phi \in E$ may be written $\phi=\left(\phi_{i j}\right)_{1 \leq i, j \leq n}$, where $\phi_{i j}$ is in $\operatorname{Hom}_{\mathrm{U}}\left(M_{j}, M_{i}\right)$.

Now by Fitting's lemma, any endomorphism of $M_{i}$ is either an automorphism or is nilpotent. With the notation $E_{i}:=\operatorname{End}_{\mathrm{U}}\left(M_{i}\right)$, it follows that for each $i$, the set $R_{i}:=\left\{\psi \in E_{i} \mid \psi\right.$ is not an automorphism $\}$ is a nilpotent ideal of $E_{i}$. In particular there is an integer $N_{i}$ such that $R_{i}^{N_{i}}=0$.

Next, suppose that we have a sequence $i=i_{1}, i_{2}, \ldots, i_{p+1}=i$, and $\phi_{j}:=$ $\phi_{i_{j}, i_{j+1}} \in \operatorname{Hom}_{\mathrm{U}}\left(M_{i_{j+1}}, M_{i_{j}}\right)$ for $j=1,2, \ldots, p$. Consider $\psi_{1}:=\phi_{1} \ldots \phi_{p-1} \phi_{p}$ in $\operatorname{Hom}_{\mathrm{U}}\left(M_{i}, M_{i}\right)$. We shall show that:

\section{$\psi_{1}$ is an automorphism $\Longrightarrow$}

the $M_{i_{j}}$ are all isomorphic, and $\phi_{j}$ is an isomorphism for each $j$.

To see (5-3), let $\psi_{j}=\phi_{j} \ldots \phi_{p} \phi_{1} \ldots \phi_{j-1} \in \operatorname{Hom}\left(M_{i_{j}}, M_{i_{j}}\right)$. If $\psi_{j}$ is an automorphism for each $j$, then for each $j, \phi_{j-1}$ is injective and $\phi_{j}$ is surjective, whence each $\phi_{j}$ is an automorphism, and we are done. If not, then there is some $j$ such that $\psi_{j}$ is nilpotent. It follows that $\psi_{1}^{N}=0$ for large $N$, which is a contradiction. This proves (5-3).

Now let $J$ be the subspace of $E$ consisting of the endomorphisms $\phi$ such that $\phi_{i j}$ is not invertible for each pair $i, j$. If

$$
J_{i j}:=\left\{\phi_{i j} \in \operatorname{Hom}_{\mathrm{U}}\left(M_{j}, M_{i}\right) \mid \phi_{i j} \text { is not invertible }\right\},
$$

then again by Fitting's lemma, $J_{i j}$ is an $\left(E_{i}, E_{j}\right)$ bimodule, and using the observation (5-3) above, it is clear that $J$ is an ideal of $E$. We shall show that $J$ is nilpotent.

Let $\phi^{(1)}, \ldots, \phi^{(\ell)}$ be a sequence of elements of $J$. Then

$$
\left(\phi^{(1)} \ldots \phi^{(\ell)}\right)_{i j}=\sum_{k_{1}, k_{2}, \ldots, k_{\ell-1}} \phi_{i k_{1}}^{(1)} \phi_{k_{1} k_{2}}^{(2)} \cdots \phi_{k_{\ell-1} j}^{(\ell)},
$$


where the sum is over all sequences $k_{1}, k_{2}, \ldots, k_{\ell-1}$ with $1 \leq k_{i} \leq n$ for all $i$.

Now we have seen that for any $j$, if $R_{j}=\operatorname{Rad} E_{j}$, then there is an integer $N_{j}$ such that $R_{j}^{N_{j}}=0$. If we take $\ell \geq N_{1}+N_{2}+\cdots+N_{n}+2$, then there some index $a$ that occurs among the $k_{i}$ at least $N_{a}+1$ times. Then each summand in the expression for $\left(\phi^{(1)} \ldots \phi^{(\ell)}\right)_{i j}$ contains a product of $N_{a}$ noninvertible elements of $E_{a}$ for some $a$, and hence is 0 . Thus $J^{N_{1}+\cdots+N_{n}+2}=0$.

Finally, it is clear that since $E_{i} / R_{i} \simeq k$ for each $i, E / J \simeq \bigoplus_{i=1}^{n} M_{d_{i}}(k)$.

The proof above actually yields the following corollary of the Artin-Wedderburn theorem.

Corollary 5.6. Let $M$ be as in Theorem 5.5 but drop the assumption on the endomorphism rings of direct summands of $M$. Then there are division rings $D_{i}$ over $k$ such that

$$
\frac{E}{\operatorname{Rad} E} \simeq \bigoplus_{i} M_{d_{i}}\left(D_{i}\right)
$$

Proof. In this case Fitting's lemma yields that $E_{i} / R_{i}$ is a division algebra $D_{i}$ over $k$, and the argument above proves the assertion.

The application to our situation arises through the following property of finitedimensional tilting modules for quantum groups. Let $k$ be a field considered as an $A$-algebra via $q \mapsto \zeta \in k \backslash\{0\}$ and let $\mathrm{U}_{\zeta}$ be as in Section 5A.

Proposition 5.7. Let $M$ be a finite-dimensional indecomposable tilting module for $\mathrm{U}_{\zeta}$ and set $E=\operatorname{End}_{\mathrm{U}_{\zeta}}(M)$. Then $E / \operatorname{Rad} E \simeq k$.

Proof. By the Ringel-Donkin classification [Donkin 1993] (see [Andersen 1992] for the adaption to the quantum case) of indecomposable tilting modules we get that $M$ has a unique highest weight $\lambda \in X^{+}$and that the weight space $M_{\lambda}$ is 1-dimensional. Therefore any $\varphi \in \operatorname{End}_{\mathrm{U}_{\zeta}}(M)$ is given by a scalar $a \in k$ on $M_{\lambda}$. But then $\varphi-a \operatorname{id}_{M}$ is not an automorphism; i.e., $\varphi-a \operatorname{id}_{M} \in \operatorname{Rad} E$.

We denote the indecomposable tilting module for $\mathrm{U}_{\zeta}$ with highest weight $\lambda$ by $\mathscr{T}_{\zeta}(\lambda)$ and for an arbitrary tilting module $\mathscr{T}$ for $U_{\zeta}$ we write $\left(\mathscr{T}: \mathscr{T}_{\zeta}(\lambda)\right)$ for the multiplicity with which $\mathscr{T}_{\zeta}(\lambda)$ occurs as a summand of $\mathscr{T}$. Then Theorem 5.5 together with Proposition 5.7 give the following result.

Corollary 5.8. For any tilting module $\mathcal{T}$ for $\mathrm{U}_{\zeta}$ and any $\lambda \in X^{+}$we have

$$
\left(\mathscr{T}: \mathscr{T}_{\zeta}(\lambda)\right)=\operatorname{dim}_{k} L_{\zeta}(\lambda)
$$

where $L_{\zeta}(\lambda)$ is the simple module for $E=\operatorname{End}_{U_{\zeta}}(\mathscr{T})$ corresponding to $\lambda$. 
5C. Multiplicities for $\mathbf{U}_{\zeta}\left(\mathfrak{s l}_{2}\right)$. We now apply the above general results to $\mathfrak{s l}_{2}$. With $k$ and $\zeta$ as above, the indecomposable tilting modules in this case are $\mathscr{T}_{\zeta}(m)$ with $m \in \mathbb{N}$. If $\zeta$ is not a root of unity in $k$ then the category of finite-dimensional $\mathrm{U}_{\zeta}$-modules is semisimple and behaves exactly like the corresponding category for the generic quantum group $\mathrm{U}_{q}$.

From now on we assume that $\zeta$ is a root of unity; for the specialisation $\mathrm{U}_{\zeta}$, etc., we assume that the homomorphism $A \rightarrow k$ is given by $q \mapsto \zeta$ (so $q^{1 / 2} \mapsto \sqrt{\zeta}$ ) and we set $\ell=\operatorname{ord}\left(\zeta^{2}\right)$. If $d$ is a positive integer with $d<\ell$ we have $\Delta_{\zeta}(d)=\mathscr{T}_{\zeta}(d)$ and all the tensor powers $\mathscr{T}_{r}=\Delta_{\zeta}(d)^{\otimes r}$ are also tilting modules. We set $E_{\zeta}(d, r)=\operatorname{End}_{\mathrm{U}_{\zeta}}\left(\mathscr{T}_{r}\right)$. By Lemma 5.1 we have

$$
E_{\zeta}(d, r)=E_{r}(d, \tilde{A}) \otimes_{\tilde{A}} k,
$$

where as before $\tilde{A}=A\left[([d] !)^{-1}\right]$. Note that our assumption $\ell>d$ ensures that the specialization $\phi_{\zeta}: A \rightarrow k$ factors through $\tilde{A}$ making $k$ into an $\tilde{A}$-algebra.

Our cellularity results from Section 3 imply that

$$
E_{\zeta}(d, r) \cong p_{\zeta} \mathrm{TL}_{d r}(k) p_{\zeta},
$$

where $p_{\zeta}$ is the specialisation at $q=\zeta$ of the idempotent $p \in \mathrm{TL}_{d r}(\tilde{A})$. Note that in $\mathrm{TL}_{d r}(k)=\mathrm{TL}_{d r, \zeta}(k)$ the generators $f_{i}$ satisfy $f_{i}^{2}=\left(\zeta+\zeta^{-1}\right) f_{i}$.

The simple modules for the cellular algebra $p_{\zeta} \mathrm{TL}_{d r}(k) p_{\zeta}$ are parametrised by the poset $\Lambda=\{m \in \mathbb{Z} \mid 0 \leq m \leq d r$ and $d r-m \in 2 \mathbb{Z}\}$; see Section 4B. We denote the simple module associated with $m \in \Lambda$ by $L_{\zeta}(m)$.

Theorem 5.9. In the above notation, in particular assuming $\ell=\operatorname{ord}\left(\zeta^{2}\right)>d$, we have for $m \in \Lambda$,

$$
\left(\mathscr{T}_{r}: \mathscr{T}_{\zeta}(m)\right)=\operatorname{dim}_{k} L_{\zeta}(m) .
$$

This multiplicity is the rank of the matrix whose rows and columns are labelled by $\mathscr{B}(d, r ; m)$ (see Section $4 A)$ and whose $\left(D_{1}, D_{2}\right)$-entry is the coefficient of the identity map $m \rightarrow m$ (in the Temperley-Lieb category) in the expansion of $D_{2}^{*} p_{\zeta} D_{1}$ as a linear combination of diagrams from $m$ to $m$.

Proof. The equality in the theorem is an immediate consequence of Corollary 5.8. To see the second statement note that $L_{\zeta}(m)$ is realised as follows: Let $W_{\zeta}(m)$ be the cell module corresponding to $m$. This has $k$-basis $C_{S}, S \in \mathscr{B}(d, r ; m)$, the monic diagrams $D$ from $m$ to $d r$ such that $L(D) \subseteq\{d, 2 d, \ldots,(r-1) d\}$. We may think of $C_{S}$ as $p_{\zeta} S$, and then the $E_{\zeta}(d, r)$-action is by left composition: for $x \in E_{\zeta}(d, r)$, $x C_{S}=\sum_{T \in \mathscr{B}(d, r ; m)} a(T, S) C_{T}$, where

$$
x p_{\zeta} S=\sum_{T \in \mathscr{B}(d, r ; m)} a(T, D) p_{\zeta} T+\text { lower terms }
$$

where "lower" means "having fewer through-arcs". 
There is an invariant form $(-,-)$ on $W_{\zeta}(m)$, defined by

$$
C_{S, T}^{m}{ }^{2} \in\left(C_{S}, C_{T}\right) C_{S, T}^{m}+E_{\zeta}(d, r)(<m) \text { for } S, T \text { in } \mathscr{B}(d, r ; m) .
$$

The radical $\operatorname{Rad}_{\zeta}(m)$ of this form is a submodule of $W_{\zeta}(m)$, and

$$
L_{\zeta}(m)=W_{\zeta}(m) / \operatorname{Rad}_{\zeta}(M) \text {. }
$$

It is therefore evident that $\operatorname{dim} L_{\zeta}(m)$ is equal to the rank of the Gram matrix $M_{m, \zeta}$, whose rows and columns are indexed by $\mathscr{B}(d, r ; m)$, and whose $(S, T)$-entry is $\left(C_{S}, C_{T}\right)$.

Finally, since $C_{S, T}^{m}{ }^{2}=p_{\zeta} S\left(T^{*} p_{\zeta} S\right) T^{*} p_{\zeta}$, and noting that $T^{*} p_{\zeta} S$ is a linear combination of diagrams from $m$ to $m$, it follows from (5-5) that $\left(C_{S}, C_{T}\right)$ is the coefficient of id $: m \rightarrow m$.

Since $\operatorname{dim} W_{\zeta}(d r)=1$ and the coefficient of id $: d \rightarrow d$ in $p_{d}(\zeta)$ is 1 , it is immediate from the theorem that the multiplicity of $\mathscr{T}_{\zeta}(d r)$ is 1 . We finish this section with a less trivial example.

Example 5.10. Take $k=d r-2$ and recall that $d<\ell$. We shall compute the multiplicity of $\mathscr{T}_{\zeta}(k)$ in $\Delta_{\zeta}(d)^{\otimes r}$ for any $d, r$. Here $\mathscr{B}(d, r ; d r-2)=\left\{S_{1}, S_{2}, \ldots, S_{r-1}\right\}$, where $S_{i}$ is as shown in the figure:

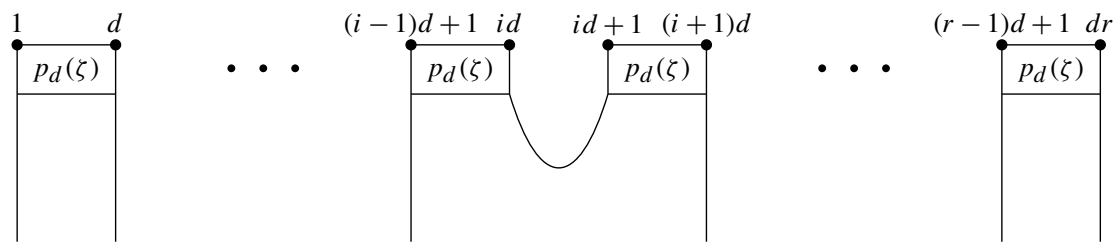

Now by repeated use of the diagrammatic recursion

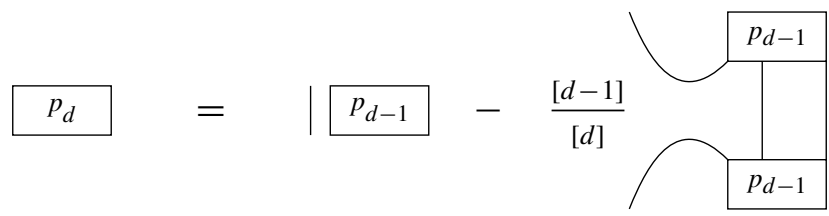

it is straightforward to compute the Gram matrix $M_{d r-2, \zeta}$ of the invariant form (see the proof above). One shows that

$$
\left(S_{i}, S_{j}\right)= \begin{cases}0 & \text { if } j \neq i \text { or } i \pm 1 \\ \frac{[2]_{\zeta d}}{[d]_{\zeta}} & \text { if } j=i \\ (-1)^{d+1}[d]_{\zeta}^{-1} & \text { if } j=i \pm 1\end{cases}
$$


Hence the Gram matrix of the invariant form is the $(r-1) \times(r-1)$ matrix

$$
M_{d r-2, \zeta}=\frac{1}{[d]_{\zeta}}\left(\begin{array}{cccccc}
\delta & (-1)^{d+1} & 0 & \cdots & \cdots & 0 \\
(-1)^{d+1} & \delta & (-1)^{d+1} & 0 & \cdots & \vdots \\
0 & (-1)^{d+1} & \delta & (-1)^{d+1} & 0 & \vdots \\
\vdots & & \ddots & \ddots & \ddots & \\
& & & & \ddots & (-1)^{d+1} \\
0 & \cdots & \cdots & 0 & (-1)^{d+1} & \delta
\end{array}\right) \text {, }
$$

where $\delta=\zeta^{d}+\zeta^{-d}=[2]_{\zeta^{d}}$.

Now it is easily shown by induction that any $n \times n$ matrix of the form

$$
A=\left(\begin{array}{cccccc}
a_{1} & b_{1} & 0 & \cdots & \cdots & 0 \\
1 & a_{2} & b_{2} & 0 & \cdots & \vdots \\
0 & 1 & a_{3} & b_{3} & 0 & \vdots \\
\vdots & & \ddots & \ddots & \ddots & \\
& & & & \ddots & b_{n-1} \\
0 & \cdots & \cdots & 0 & 1 & a_{n}
\end{array}\right)
$$

with entries in a principal ideal domain may be transformed by row and column operations into

$$
A^{\prime}=\left(\begin{array}{cccccc}
1 & 0 & 0 & \cdots & \cdots & 0 \\
0 & 1 & 0 & 0 & \cdots & \vdots \\
0 & 0 & 1 & 0 & 0 & \vdots \\
\vdots & & \ddots & \ddots & \ddots & \\
& & & \ddots & 1 & 0 \\
0 & \cdots & \cdots & 0 & 0 & D
\end{array}\right)
$$

where $D=\operatorname{det}(A)$. It follows that the rank of the Gram matrix $M_{d r-2, \zeta}$ is $r-1$ if $\operatorname{det} M_{d r-2, \zeta} \neq 0$, while if det $M_{d r-2, \zeta}=0$, the rank is $r-2$.

Now the determinant of $[d]_{\zeta} M_{d r-2, \zeta}$ is easily computed (cf. [Graham and Lehrer 1996, Equation 6.18.2]), and using this, we see that

$$
\operatorname{det} M_{d r-2, \zeta}=(-1)^{(d+1)(r+1)}\left([d]_{\zeta}\right)^{-(r-1)}[r]_{(-1)^{d+1} \zeta^{d}} .
$$

It therefore follows that the multiplicity of $\mathscr{T}_{\zeta}(d r-2)$ in $\Delta_{\zeta}(d)^{\otimes r}$ is

$$
\begin{cases}r-1 & \text { if }[r]_{(-1)^{d+1} \zeta^{d}} \neq 0 \\ r-2 & \text { otherwise. }\end{cases}
$$


Finally, observe that

$$
[r]_{(-1)^{d+1} \zeta^{d}}=0 \quad \Longleftrightarrow \quad \zeta^{2 d r}=1 .
$$

Hence if we write (using the convention that for any root of unity $\xi$, we denote by $|\xi|$ or by $\operatorname{ord}(\xi)$ the multiplicative order of $\xi$ )

$$
\ell= \begin{cases}|\zeta| & \text { if }|\zeta| \text { is odd } \\ \frac{1}{2}|\zeta| & \text { if }|\zeta| \text { is even, }\end{cases}
$$

then $\ell=\left|\zeta^{2}\right|$, whence the multiplicity of $\mathscr{T}_{\zeta}(d r-2)$ in $\Delta_{\zeta}(d)^{\otimes r}$ is given by

$$
\left(\mathscr{T}_{r}: \mathscr{T}_{\zeta}(d r-2)\right)= \begin{cases}r-1 & \text { if } \ell \nmid d r \\ r-2 & \text { if } \ell \mid d r .\end{cases}
$$

This shows also by standard cellular theory that the cell module $W_{\zeta}(d r-2)$ of $\left.E_{\zeta}(d, r)\right)$ is simple if $\ell \nmid d r$, while if $\ell \mid d r$, then $W_{\zeta}(d r-2)$ has composition factors $L_{\zeta}(d, r ; d r-2)$ and $L_{\zeta}(d, r ; d r)$ (the latter being the trivial module), each with multiplicity one.

\section{Complex roots of unity}

In this section we take $k=\mathbb{C}$ and fix a root of unity $\zeta \in \mathbb{C}$. As before we set $\ell=$ $\operatorname{ord}\left(\zeta^{2}\right)$. In this case the structure of the tilting modules $\mathscr{T}_{\zeta}(m)$ is well understood, and hence, when $\ell>d$, provides an alternative approach to the computation of the multiplicities $\mu_{\zeta}(d, r ; m):=\left(\Delta_{\zeta}(d)^{\otimes r}: \mathscr{T}_{\zeta}(m)\right)$, and thus of the dimensions of the simple modules for the cellular algebra $E_{\zeta}(d, r)$ (see Theorem 5.9). In this section we demonstrate how this is done. We then show how these results on tilting modules may alternatively be deduced from results on the decomposition numbers of the algebras $E_{\zeta}(d, r)$, which are also proved in this section.

\section{A. Structure of tilting modules.}

Proposition 6.1. The indecomposable tilting module $\mathscr{T}_{\zeta}(m)$ for $\mathrm{U}_{\zeta}=\mathrm{U}_{\zeta}\left(\mathfrak{s l}_{2}\right)$ with highest weight $m$ has the following description.

(1) If either $m<\ell$ or $m \equiv-1(\bmod \ell)$ then $\mathscr{T}_{\zeta}(m) \simeq \Delta_{\zeta}(m)$ is irreducible.

(2) Write $m=a \ell+b$, where $a \geq 1$ and $0 \leq b<\ell-1$. Then $\mathscr{T}_{\zeta}(m)$ is the unique nontrivial extension

$$
0 \longrightarrow \Delta_{\zeta}(m) \longrightarrow \mathscr{T}_{\zeta}(m) \longrightarrow \Delta_{\zeta}(m-2 b-2) \longrightarrow 0 .
$$

Proof. This result is certainly well known and follows from the results of [Soergel 1998]. As we haven't been able to find a reference where this is explicitly stated we sketch the easy proof. 
Denote by $\mathscr{L}_{\zeta}(m)$ the simple $\mathrm{U}_{\zeta}$-module with highest weight $m \in \mathbb{N}$ (not to be confused with the simple $E_{\zeta}(d, r)$-module $L_{\zeta}(m)$ ). It follows from the strong linkage principle [Andersen 2003] (or by direct calculations) that $\mathscr{L}_{\zeta}(m)=\Delta_{\zeta}(m)$ if and only if $m$ satisfies the conditions in (1); in particular, (1) holds.

So assume $m=a \ell+b$ with $a$ and $b$ as in (2). The module $\Delta(a \ell-1) \otimes_{\mathbb{C}} \Delta_{\zeta}(b+1)$ has a Weyl filtration with factors $\Delta_{\zeta}(m), \Delta_{\zeta}(m-2), \cdots, \Delta_{\zeta}(m-2(b+1))$. Note that the first and the last factors belong to the same linkage class and that none of the other factors are in this class. Hence by the linkage principle [loc. cit.] there is a summand $\mathscr{T}$ of $\Delta_{\zeta}(a \ell-1) \otimes_{\mathbb{C}} \Delta_{\zeta}(b+1)$ which has these two Weyl factors, i.e., fits into an exact sequence

$$
0 \longrightarrow \Delta_{\zeta}(m) \longrightarrow \mathcal{T} \longrightarrow \Delta_{\zeta}(m-2 b-2) \longrightarrow 0 .
$$

By case (1) we see that $\Delta_{\zeta}(a \ell-1) \otimes_{\mathbb{C}} \Delta_{\zeta}(b+1)$ is tilting. Hence so is our summand $\mathscr{T}$. The proof of case (2) will therefore be complete if we prove that $\mathscr{T}$ is indecomposable. This in turn would follow if there were no nontrivial homomorphisms $\mathscr{T}$ of $\Delta_{\zeta}(a \ell-1) \otimes_{\mathbb{C}} \Delta_{\zeta}(b+1) \longrightarrow \mathscr{L}_{\zeta}(m)$, for if the last sequence splits, there would be such a homomorphism. To check the last statement, we need the quantised Steinberg tensor product theorem [Andersen and Wen 1992, Theorem 1.10] for simple modules, $\mathscr{L}_{\zeta}(m) \simeq \mathscr{L}_{\zeta}(a \ell) \otimes \mathscr{L}_{\zeta}(b)$ (again in the case at hand this can alternatively be checked by direct calculations).

Using this together with the self-duality of simple modules and the result in (1) we get

$$
\begin{aligned}
\operatorname{Hom}_{\mathrm{U}_{\zeta}} & \left.\Delta_{\zeta}(a \ell-1) \otimes_{\mathbb{C}} \Delta_{\zeta}(b+1), \mathscr{L}_{\zeta}(m)\right) \\
& \simeq \operatorname{Hom}_{\mathrm{U}_{\zeta}}\left(\mathscr{L}_{\zeta}(a \ell-1) \otimes_{\mathbb{C}} \mathscr{L}_{\zeta}(b+1), \mathscr{L}_{\zeta}(m)\right) \\
& \simeq \operatorname{Hom}_{\mathrm{U}_{\zeta}}\left(\mathscr{L}_{\zeta}((a-1) \ell) \otimes_{\mathbb{C}} \mathscr{L}_{\zeta}(\ell-1) \otimes_{\mathbb{C}} \mathscr{L}_{\zeta}(b+1), \mathscr{L}_{\zeta}(a \ell) \otimes_{\mathbb{C}} \mathscr{L}_{\zeta}(b)\right) \\
& \simeq \operatorname{Hom}_{\mathrm{U}_{\zeta}}\left(\mathscr{L}_{\zeta}((a-1) \ell) \otimes_{\mathbb{C}} \mathscr{L}_{\zeta}(b+1) \otimes_{\mathbb{C}} \mathscr{L}_{\zeta}(b), \mathscr{L}_{\zeta}(a \ell) \otimes_{\mathbb{C}} \mathscr{L}_{\zeta}(\ell-1)\right) \\
& \simeq \operatorname{Hom}_{\mathrm{U}_{\zeta}}\left(\mathscr{L}_{\zeta}((a-1) \ell) \otimes_{\mathbb{C}} \mathscr{L}_{\zeta}(b+1) \otimes_{\mathbb{C}} \mathscr{L}_{\zeta}(b), \mathscr{L}_{\zeta}((a+1) \ell-1)\right) .
\end{aligned}
$$

The last Hom-space is 0 because, by our condition on $b$, the weight $(a+1) \ell-1$ is strictly larger than all weights of $\mathscr{L}_{\zeta}((a-1) \ell) \otimes_{\mathbb{C}} \mathscr{L}_{\zeta}(b+1) \otimes_{\mathbb{C}} \mathscr{L}_{\zeta}(b)$.

Since the weights of $\Delta_{\zeta}(m)$ are $m, m-2, \cdots,-m$, each occurring with multiplicity one, we deduce the following result.

Corollary 6.2. We have

$$
\operatorname{dim} \mathscr{T}_{\zeta}(m)_{t}= \begin{cases}1 & \text { if } t=m-2 i, 0 \leq i \leq m \text { in case }(1) \\ 2 & \text { if } t=m-2 j, b+1 \leq j \leq m-(b+1) \text { in case }(2) \\ 1 & \text { if } t=m-2 j, \text { with } 0 \leq j \leq b \text { or } m \geq j \geq m-b \text { in case }(2) \\ 0 & \text { otherwise. }\end{cases}
$$


6B. Multiplicities and dimensions. Now the equation

$$
\Delta_{\zeta}(d)^{\otimes r} \cong \bigoplus_{m=0}^{d r} \mu_{\zeta}(d, r ; m) \mathscr{T}_{\zeta}(m)
$$

may be used to relate the multiplicities to the dimensions of the weight spaces. For this purpose, we make the following definitions.

Definition 6.3. (1) Let $w(d, r ; m):=\operatorname{dim}\left(\Delta_{\zeta}(d)^{\otimes r}\right)_{m}$. This is independent of $\zeta$.

(2) Let $a_{m}=a_{m}(d, r):=\mid\left\{\left(i_{1}, \ldots, i_{r}\right) \mid 0 \leq i_{j} \leq d\right.$ for all $j$ and $\left.\sum_{j} i_{j}=m\right\} \mid$.

Note that $a_{m}=a_{d r-m}$ for all $m$.

Lemma 6.4. (1) For $0 \leq m \leq d r, m \equiv d r(\bmod 2), w(d, r ; m)=a_{(m+d r) / 2}$.

(2) We have

$$
w(d, r ; m)=\mu_{\zeta}(d, r ; m)+\sum_{j=1}^{(d r-m) / 2} \operatorname{dim} \mathscr{T}_{\zeta}(m+2 j)_{m} \mu_{\zeta}(d, r ; m+2 j) .
$$

The first statement follows easily from the fact that $\Delta_{\zeta}(d)^{\otimes r}$ has $q$-character $[d+1]^{r}$, while the second arises from (6-1) by taking the dimension of the $m$-weight spaces on both sides, taking into account that $\mathscr{T}_{\zeta}(t)$ has only weights $m$ that satisfy $m=t-2 i, i \geq 0$, and $r d \geq m \geq-r d$.

Lemma 6.4(2) may be used to determine the multiplicities $\mu_{\zeta}(d, r ; m)$ recursively. We shall do this for the case considered in Example 5.10.

Example 6.5. Let us compute $\mu_{\zeta}(d, r, d r-2)$. By Lemma 6.4(2),

$$
w(d, r ; d r-2)=\mu_{\zeta}(d, r ; d r-2)+\operatorname{dim} \mathscr{T}_{\zeta}(d r)_{d r-2} .
$$

Moreover, it follows from Corollary 6.2 that

$$
\operatorname{dim} \mathscr{T}_{\zeta}(d r)_{d r-2}= \begin{cases}2 & \text { if } b=0 \\ 1 & \text { if } b \neq 0\end{cases}
$$

Noting that by Lemma 6.4(1) we have $w(d, r, d r-2)=a_{d r-1}=a_{1}=r$, we get

$$
\mu_{\zeta}(d, r ; d r-2)= \begin{cases}r-1 & \text { if } \ell \nmid d r, \\ r-2 & \text { if } \ell \mid d r,\end{cases}
$$

in accord with (5-7).

Example 6.6. In Example 6.5 we considered multiplicities $\mu_{\zeta}(d, r ; t)$, where $t$ was large, namely $t=d r-2$. We now consider the case where $t$ is small.

Assume $t<\ell$. Then we may apply [Andersen and Paradowski 1995, Formula 3.20(1)]. Using the notation from Section 4A this formula reads in our case

$$
\mu_{\zeta}(d, r ; t)=\sum_{j \geq 0} m(d, r ; t+2 j \ell)-\sum_{i>0} m(d, r ; 2 i \ell-t-2) .
$$


Recall that the multiplicities $m(d, r ; t)$ are given by the recursion relation (4-8); i.e., they may be calculated by induction on $r$.

In fact this formula is valid in general: maintaining the notation of Example 6.6 (except that the integer $t$ below may now be arbitrary) we have the following result.

Proposition 6.7. Let $t \in \mathbb{N}$.

(1) If $t \equiv-1(\bmod \ell)$ then $\mu_{\zeta}(d, r ; t)=m(d, r ; t)$.

(2) If $t \not \equiv-1(\bmod \ell)$ then, writing $t=a \ell+b$ with $0 \leq b \leq \ell-2$, we have

$$
\begin{aligned}
\mu_{\zeta}(d, r ; t) & =\sum_{j \geq 0} m(d, r ; t+2 j \ell)-\sum_{i \geq 1} m(d, r ; t-2 b-2+2 i \ell) \\
& =\sum_{j \geq 0} m(d, r ; t+2 j \ell)-\sum_{i \geq a+1} m(d, r ; 2 i \ell-t-2) .
\end{aligned}
$$

Proof. This follows easily from the description of the indecomposable tilting modules $\mathscr{T}_{\zeta}(m)$ in Proposition 6.1 by taking characters in the relation $\Delta_{\zeta}(d)^{\otimes r} \cong$ $\bigoplus_{m} \mu(d, r ; m) \mathscr{T}_{\zeta}(m)$. Let $\mathscr{C}_{1}$ be the set of positive integers occurring in case (1) of Proposition 6.1, and similarly let $\mathscr{C}_{2}$ be those occurring in case (2).

If we denote by $c_{t}$ the $q$-character of $\Delta_{q}(t)$, then Proposition 6.1 shows that if $t \in \mathscr{C}_{1}$, then $\operatorname{char}\left(\mathscr{T}_{\zeta}(t)\right)=c_{t}$, while if $t \in \mathscr{C}_{2}$, then $\operatorname{char}\left(\mathscr{T}_{\zeta}(t)\right)=c_{t}+c_{t-2 b-2}$. Now substitute these values and compare coefficients of $c_{t}$ in the equation

$$
\sum_{t \in \mathbb{N}} m(d, r ; t) c_{t}=\sum_{t \in \mathscr{C}_{1}} \mu_{\zeta}(d, r ; t) \operatorname{char}\left(\mathscr{T}_{\zeta}(t)\right)+\sum_{t \in \mathscr{C}_{2}} \mu_{\zeta}(d, r ; t) \operatorname{char}\left(\mathscr{T}_{\zeta}(t)\right) .
$$

One obtains $\mu_{\zeta}(d, r ; t)=m(d, r ; t)$ if $t \equiv-1(\bmod \ell)$, while if $t=a \ell+b$ with $a \geq 0$ and $0 \leq b \leq \ell-2$, we have

$$
m(d, r ; t)=\mu_{\zeta}(d, r ; t)+\mu_{\zeta}(d, r ;(a+2) \ell-b-2) .
$$

Now for any integer $t=a \ell+b \geq 0$ such that $t \not \equiv-1(\bmod \ell)$, write $g(t)=$ $(a+2) \ell-b-2$; then $g(t) \not \equiv-1(\bmod \ell)$, and the relation above reads $m(d, r ; t)=$ $\mu_{\zeta}(d, r ; t)+\mu_{\zeta}(d, r ; g(t))$. It follows that

$$
\mu_{\zeta}(d, r ; t)=\sum_{i \geq 0} m\left(d, r ; g^{2 i}(t)\right)-\sum_{j \geq 0} m\left(d, r ; g^{2 j+1}(t)\right) .
$$

The statements (1) and (2) are now immediate.

As these multiplicities are also dimensions of simple modules for our cellular algebra from Section 4, we may rewrite these formulae as follows (again using notation from Section 4A).

Corollary 6.8. Let $t \in \mathbb{N}$.

(1) If $t \equiv-1(\bmod \ell)$ then $\operatorname{dim}_{\mathbb{C}} L_{\zeta}(t)=b(d, r ; t)$. 
(2) If $t \not \equiv-1(\bmod \ell)$ then, writing $t=a \ell+b$ with $0 \leq b \leq \ell-2$, we have

$$
\operatorname{dim}_{\mathbb{C}} L_{\zeta}(t)=\sum_{j \geq 0} b(d, r ; t+2 j \ell)-\sum_{i \geq a+1} b(d, r ; 2 i \ell-t-2) .
$$

Note that the numbers $b(d, r ; t)$ are dimensions of the cell modules of the cellular algebra $p \mathrm{TL}_{d r}(\tilde{A}) p$ that do not change under specialisation.

6C. Decomposition numbers. In this section we shall determine the decomposition numbers of the cellular algebra $E_{\zeta}(d, r)$, and show how the weight multiplicities of the tilting modules are determined by these, giving an alternative proof of Corollary 6.2. The algebra has cell modules $W_{\zeta}(t)$ as implied in Section 4B and $\operatorname{dim}\left(W_{\zeta}(t)\right)=b(d, r ; t)$. If $L_{\zeta}(t)$ is the corresponding simple module, we write $d_{s t}=\left[W_{\zeta}(t): L_{\zeta}(s)\right]$ for the multiplicity of $L_{\zeta}(s)$ in $W_{\zeta}(t)$. It is known by the theory of cellular algebras that the matrix $\left(d_{s t}\right)$ is lower unitriangular.

We have $\operatorname{dim}\left(L_{\zeta}(t)\right)=\mu_{\zeta}(d, r ; t)$, and therefore we clearly have

$$
b(d, r ; t)=\sum_{s \geq t} d_{s t} \mu_{\zeta}(d, r ; s) .
$$

Theorem 6.9. Maintain the notation above. Suppose $\ell \in \mathbb{N}$ is such that $\ell=\operatorname{ord}\left(\zeta^{2}\right)$ and $\ell>d$, and write $\mathbb{N}=\mathcal{N}_{1} \amalg \mathcal{N}_{2}$, where $\mathcal{N}_{1}=\{t \in \mathbb{N} \mid t \equiv-1(\bmod \ell)\}$ and $\mathcal{N}_{2}=\mathbb{N} \backslash \mathcal{N}_{1}$. Let $g: \mathcal{N}_{2} \longrightarrow \mathcal{N}_{2}$ be the function defined in the proof of Proposition 6.7, viz. if $t=a \ell+b$ with $0 \leq b \leq \ell-2$, then $g(t)=(a+1) \ell+\ell-b-2$. Observe that $g(t)=t+2(\ell-b-1) \geq t+2$, and that $g(t) \equiv t(\bmod 2)$.

(1) For each $t \in \mathcal{N}_{2}$ such that $0 \leq t<g(t) \leq d r$ and $t \equiv d r(\bmod 2)$, there is a nonzero homomorphism $\theta_{t}: W_{\zeta}(g(t)) \longrightarrow W_{\zeta}(t)$ which is uniquely determined up to scalar multiplication.

(2) The $\theta_{t}$ are the only nontrivial homomorphisms between the cell modules of $E_{\zeta}(d, r)$.

(3) Let $t \in \mathbb{N}$ be such that $0 \leq t \leq d r$ and $t \equiv d r(\bmod 2)$. If $t \in \mathcal{N}_{2}$ and $g(t) \leq d r$, then $W_{\zeta}(t)$ has composition factors $L_{\zeta}(t)$ and $L_{\zeta}(g(t))$, each with multiplicity 1 . All other cell modules are simple.

(4) The decomposition numbers of $E_{\zeta}(d, r)$ are all equal to 0 or 1.

Note that (3) and (4) are formal consequences of (1) and (2).

Proof. We begin by observing that the statement is true when $d=1$. In this case $E_{\zeta}(1, r)=\mathrm{TL}_{r, \zeta}(\mathbb{C})$, the structure of whose cell modules (as well as all homomorphisms between them) is treated in [Graham and Lehrer 1998]. In particular, Theorem 5.3 of that reference asserts that (in our notation above) if $s \neq t$, then $L_{\zeta}(s)$ is a composition factor of $W_{\zeta}(t)$ if and only if $s$ satisfies both (i) $t+2 \ell>s>t$ 
and (ii) $s+t+2 \equiv 0(\bmod 2 \ell)$. It is an easy exercise to show that (i) and (ii) are equivalent to (iii) $t \not \equiv-1(\bmod \ell)$ and (iv) $s=g(t)$. This yields all the statements of the theorem for this case.

Next recall that $E_{\zeta}(d, r) \cong p_{d}(\zeta) \mathrm{TL}_{d r, \zeta}(\mathbb{C}) p_{d}(\zeta)$, where $p_{d}(\zeta)$ is the specialisation at $\zeta$ of the idempotent $p_{d}$. Thus we may define the exact functor $\mathscr{F}_{d}: \operatorname{Mod}\left(\mathrm{TL}_{d r, \zeta}(\mathbb{C})\right) \longrightarrow \operatorname{Mod}\left(E_{\zeta}(d, r)\right)$ by $M \mapsto p_{d}(\zeta) M$, where Mod indicates the category of left modules for the relevant algebra. Now it is evident from the description in Section $4 \mathrm{~B}$ of the cell module $W(t)$ and its basis $\mathscr{B}(d, r ; t)$ that $\mathscr{F}_{d}\left(W_{\mathrm{TL}_{d r, \zeta}(\mathbb{C})}(t)\right)=W_{E_{\zeta}(d, r)}(t)$ for all $t$ with $0 \leq t \leq d r$ and $t+d r \in 2 \mathbb{Z}$.

Moreover by exactness, for any simple $\mathrm{TL}_{d r, \zeta}(\mathbb{C})$-module $L, \mathscr{F}_{d}(L)$ is either a simple $E_{\zeta}(d, r)$-module or zero. Thus it follows (also from the explicit diagrammatic description) that $\mathscr{F}_{d}\left(L_{\mathrm{TL}_{d r, \zeta}(\mathbb{C})}(t)\right)=L_{E_{\zeta}(d, r)}(t)$ whenever the latter is nonzero. Given the description in Section $4 \mathrm{~B}$ of the cellular structure, and the fact that $\mathrm{TL}_{d r, \zeta}(\mathbb{C})$ is quasihereditary when $\zeta \neq \zeta_{4}=\exp (\pi i / 2), \mathscr{F}_{d}$ does not kill any nontrivial simple $\mathrm{TL}_{d r, \zeta}(\mathbb{C})$-module (this may be checked directly when $\zeta=\zeta_{4}$ ). The quasiheredity of $\mathrm{TL}_{d r, \zeta}(\mathbb{C})$ when $\zeta \neq \zeta_{4}$ is well known, but may be seen as follows.

Since $\zeta+\zeta^{-1} \neq 0$, if $t \in \mathbb{N}, 0 \leq t \leq d r, t \equiv d r(\bmod 2)$, then for any monic diagram $u: t \rightarrow d r$, we have $u^{*} u=\left(\zeta+\zeta^{-1}\right)^{(d r-t) / 2} \mathrm{id}_{t} \neq 0$; hence, if $u$ is thought of as an element of $W_{\zeta}(t)$, then $(u, u) \neq 0$. Thus, for any such $t, L_{\zeta}(t) \neq 0$. Although it is not needed for the proof of the theorem, the fact that if $L_{\mathrm{TL}_{d r, \zeta}(\mathbb{C})}(t) \neq 0$ then $\mathscr{F}_{d}\left(L_{\mathrm{TL}_{d r, \zeta}(\mathbb{C})}(t)\right) \neq 0$ is verified in the same way, but requires a computation, using the recurrence (5-6) in Example 5.10 above, to show that for a nonzero element $u=p_{d} D \in W_{\zeta}(t)$, where $D: t \rightarrow d r$ is a monic diagram, we have $(u, u) \neq 0$. That such elements exist is easily verified.

By the case $d=1$ of Theorem 6.9 or, more precisely, [Graham and Lehrer 1998, Theorem 5.3] applied to $\mathrm{TL}_{d r, \zeta}(\mathbb{C})$, if $t \in \mathcal{N}_{2}, 0 \leq t<g(t) \leq d r$ and $t \equiv d r(\bmod 2)$, then $W_{\mathrm{TL}_{d r, \zeta}(\mathbb{C})}(t)$ has composition factors $L_{\mathrm{TL}_{d r, \zeta}(\mathbb{C})}(t)$ and $L_{\mathrm{TL}_{d r, \zeta}(\mathbb{C})}(g(t))$. All other cell modules for $\mathrm{TL}_{d r, \zeta}(\mathbb{C})$ are simple. It follows from the previous paragraph that similarly, if $t \in \mathcal{N}_{2}, 0 \leq t<g(t) \leq d r$ and $t \equiv d r(\bmod 2)$, then $W_{E_{\zeta}(d, r)}(t)$ has composition factors $L_{E_{\zeta}(d, r)}(t)$ and $L_{E_{\zeta}(d, r)}(g(t))$, and that other cell modules for $E_{\zeta}(d, r)$ are simple. All statements in the theorem are now easy consequences of standard cellular theory.

Remark 6.10. (1) From Theorem 6.9 it follows that (6-3) implies (6-2) and the other statements in Proposition 6.7. Thus the multiplicities $\mu_{\zeta}(d, r ; t)$ are determined by Theorem 6.9.

(2) Since the dimensions $w(d, r ; t)$ are known (Lemma 6.4(1)), it follows from Lemma 6.4(2) that the dimensions of the weight spaces $\mathscr{T}_{\zeta}(d r)_{m}$ are determined by Theorem 6.9. 
(3) There are some analogies between this work and the modular theory developed by Erdmann [1995]. In the case $n=2$, Erdmann dealt only with the 2dimensional representation of $\mathfrak{g l}_{2}$. Nonetheless, there appear to be some similarities between her formulae and the Gram determinants of the cell modules in our situation.

\section{Acknowledgements}

The present work was initiated during a visit by Prof. Andersen to the University of Sydney in October 2012 and he gratefully acknowledges the support and hospitality extended to him there. He was also supported by the Danish National Research Foundation Center of Excellence, Centre for Quantum Geometry of Moduli Spaces (QGM).

Prof. Lehrer and Prof. Zhang were supported by the Australian Research Council, through Discovery Projects DP120103432 (Quantised algebras, supersymmetry and invariant theory) and DP0772870 (Invariant theory, cellularity and geometry).

\section{References}

[Andersen 1992] H. H. Andersen, "Tensor products of quantized tilting modules", Comm. Math. Phys. 149:1 (1992), 149-159. MR 94b:17015 Zbl 0760.17004

[Andersen 2003] H. H. Andersen, "The strong linkage principle for quantum groups at roots of 1", $J$. Algebra 260:1 (2003), 2-15. MR 2004c:17024 Zbl 1043.17005

[Andersen 2012] T. S. Andersen, Endomorphism algebras of tensor powers of modules for quantum groups, thesis, Aarhus University, 2012, Available at http://math.au.dk/publs?publid=953.

[Andersen and Paradowski 1995] H. H. Andersen and J. Paradowski, "Fusion categories arising from semisimple Lie algebras”, Comm. Math. Phys. 169:3 (1995), 563-588. MR 96e:17026 Zbl 0827.17010

[Andersen and Wen 1992] H. H. Andersen and K. Wen, "Representations of quantum algebras: the mixed case”, J. Reine Angew. Math. 427 (1992), 35-50. MR 93j:17019 Zbl 0771.17010

[Andersen et al. 2008] H. H. Andersen, G. I. Lehrer, and R. B. Zhang, "Endomorphism algebras of tensor modules for quantum groups at roots of unity", unfinished manuscript (available from the authors), 2008.

[Brundan and Kleshchev 1999] J. Brundan and A. Kleshchev, "Modular Littlewood-Richardson coefficients", Math. Z. 232:2 (1999), 287-320. MR 2000i:20071 Zbl 0945.20027

[Chari and Pressley 1994] V. Chari and A. Pressley, A guide to quantum groups, Cambridge University Press, 1994. MR 95j:17010 Zbl 0839.17009

[Donkin 1993] S. Donkin, "On tilting modules for algebraic groups", Math. Z. 212:1 (1993), 39-60. MR 94b:20045 Zbl 0798.20035

[Drinfeld 1987] V. G. Drinfeld, "Quantum groups", pp. 798-820 in Proceedings of the International Congress of Mathematicians (Berkeley, CA, 1986), vol. 1, edited by A. M. Gleason, American Mathematical Society, Providence, RI, 1987. MR 89f:17017 Zbl 0667.16003 
[Du et al. 1998] J. Du, B. Parshall, and L. Scott, "Quantum Weyl reciprocity and tilting modules", Comm. Math. Phys. 195:2 (1998), 321-352. MR 99k:17026 Zbl 0936.16008

[Erdmann 1995] K. Erdmann, "Tensor products and dimensions of simple modules for symmetric groups”, Manuscripta Math. 88:3 (1995), 357-386. MR 96j:20018 Zbl 0849.20008

[Fan and Green 1997] C. K. Fan and R. M. Green, "Monomials and Temperley-Lieb algebras", J. Algebra 190:2 (1997), 498-517. MR 98a:20037 Zbl 0899.20018

[Graham and Lehrer 1996] J. J. Graham and G. I. Lehrer, "Cellular algebras", Invent. Math. 123:1 (1996), 1-34. MR 97h:20016 Zbl 0853.20029

[Graham and Lehrer 1998] J. J. Graham and G. I. Lehrer, "The representation theory of affine Temperley-Lieb algebras”, Enseign. Math. (2) 44 (1998), 173-218. MR 99i:20019 Zbl 0964.20002

[Graham and Lehrer 2003] J. J. Graham and G. I. Lehrer, "Diagram algebras, Hecke algebras and decomposition numbers at roots of unity", Ann. Sci. École Norm. Sup. (4) 36:4 (2003), 479-524. MR 2004k:20007 Zbl 1062.20003

[Graham and Lehrer 2004] J. J. Graham and G. I. Lehrer, "Cellular algebras and diagram algebras in representation theory", pp. 141-173 in Representation theory of algebraic groups and quantum groups (Tokyo, 2001), edited by T. Shoji et al., Advanced Studies in Pure Mathematics 40, Mathematical Society of Japan, Tokyo, 2004. MR 2005i:20005 Zbl 1135.20302

[Jantzen 2003] J. C. Jantzen, Representations of algebraic groups, 2nd ed., Mathematical Surveys and Monographs 107, American Mathematical Society, Providence, RI, 2003. MR 2004h:20061 Zbl 1034.20041

[Jimbo 1986] M. Jimbo, "A $q$-analogue of $U(\mathfrak{g l}(N+1))$, Hecke algebra, and the Yang-Baxter equation”, Lett. Math. Phys. 11:3 (1986), 247-252. MR 87k:17011 Zbl 0602.17005

[Kirillov and Reshetikhin 1990] A. N. Kirillov and N. Reshetikhin, " $q$-Weyl group and a multiplicative formula for universal $R$-matrices”, Comm. Math. Phys. 134:2 (1990), 421-431. MR 92c:17023 Zbl 0723.17014

[Lehrer and Zhang 2006] G. I. Lehrer and R. B. Zhang, "Strongly multiplicity free modules for Lie algebras and quantum groups", J. Algebra 306:1 (2006), 138-174. MR 2007g:17008 Zbl 1169.17003

[Lehrer and Zhang 2010] G. I. Lehrer and R. B. Zhang, "A Temperley-Lieb analogue for the BMV algebra", pp. 155-190 in Representation theory of algebraic groups and quantum groups (Nagoya, 2006), edited by A. Gyoja et al., Progress in Mathematics 284, Birkhäuser, New York, 2010. MR 2012b:16098 Zbl 1260.16030

[Lusztig 1988] G. Lusztig, "Quantum deformations of certain simple modules over enveloping algebras", Adv. in Math. 70:2 (1988), 237-249. MR 89k:17029 Zbl 0651.17007

[Lusztig 1990] G. Lusztig, "Quantum groups at roots of 1", Geom. Dedicata 35:1-3 (1990), 89-113. MR 91j:17018 Zbl 0714.17013

[Lusztig 1993] G. Lusztig, Introduction to quantum groups, Progress in Mathematics 110, Birkhäuser, Boston, 1993. MR 94m:17016 Zbl 0788.17010

[Mathieu and Papadopoulos 1999] O. Mathieu and G. Papadopoulos, "A character formula for a family of simple modular representations of GL $n$ ”, Comment. Math. Helv. 74:2 (1999), 280-296. MR 2000f:20012 Zbl 0935.20029

[Ryom-Hansen 2003] S. Ryom-Hansen, "A q-analogue of Kempf's vanishing theorem", Mosc. Math. J. 3:1 (2003), 173-187. MR 2004e:17014 Zbl 1062.17013

[Soergel 1998] W. Soergel, "Character formulas for tilting modules over Kac-Moody algebras", Represent. Theory 2 (1998), 432-448. MR 2000c:17048 Zbl 0964.17018 
Received July 3, 2014. Revised October 25, 2014.

HENNING H. ANDERSEN

QGM, Det NATURVIDEnskabelige FAKUltet

AARHUS UNIVERSITET

NY MUNKEGADE BYGNING 1530

DK-8000 AARHUS

DENMARK

mathha@imf.au.dk

GUSTAV I. LEHRER

SCHOOL OF MATHEMATICS AND STATISTICS F07

UNIVERSITY OF SYDNEY

NSW 2006

SYDNEY 2006

Australia

gustav.lehrer@sydney.edu.au

RUIBIN ZHANG

SCHOOL OF MATHEMATICS AND STATISTICS

UNIVERSITY OF SYDNEY

N.S.W. 2006

SYDNEY 2006

AUSTRALIA

ruibin.zhang@sydney.edu.au 


\title{
PACIFIC JOURNAL OF MATHEMATICS
}

\author{
msp.org/pjm
}

Founded in 1951 by E. F. Beckenbach (1906-1982) and F. Wolf (1904-1989)

\section{EDITORS}

Don Blasius (Managing Editor)

Department of Mathematics

University of California

Los Angeles, CA 90095-1555

blasius@math.ucla.edu

\author{
Paul Balmer \\ Department of Mathematics \\ University of California \\ Los Angeles, CA 90095-1555 \\ balmer@math.ucla.edu \\ Robert Finn \\ Department of Mathematics \\ Stanford University \\ Stanford, CA 94305-2125 \\ finn@math.stanford.edu \\ Sorin Popa \\ Department of Mathematics \\ University of California \\ Los Angeles, CA 90095-1555 \\ popa@math.ucla.edu
}

\author{
Vyjayanthi Chari \\ Department of Mathematics \\ University of California \\ Riverside, CA 92521-0135 \\ chari@math.ucr.edu \\ Kefeng Liu \\ Department of Mathematics \\ University of California \\ Los Angeles, CA 90095-1555 \\ liu@math.ucla.edu \\ Jie Qing \\ Department of Mathematics \\ University of California \\ Santa Cruz, CA 95064 \\ qing@ cats.ucsc.edu
}

\section{PRODUCTION}

Silvio Levy, Scientific Editor, production@msp.org

\section{SUPPORTING INSTITUTIONS}

ACADEMIA SINICA, TAIPEI

CALIFORNIA INST. OF TECHNOLOGY

INST. DE MATEMÁTICA PURA E APLICADA

KEIO UNIVERSITY

MATH. SCIENCES RESEARCH INSTITUTE

NEW MEXICO STATE UNIV.

OREGON STATE UNIV.

\author{
STANFORD UNIVERSITY \\ UNIV. OF BRITISH COLUMBIA \\ UNIV. OF CALIFORNIA, BERKELEY \\ UNIV. OF CALIFORNIA, DAVIS \\ UNIV. OF CALIFORNIA, LOS ANGELES \\ UNIV. OF CALIFORNIA, RIVERSIDE \\ UNIV. OF CALIFORNIA, SAN DIEGO \\ UNIV. OF CALIF., SANTA BARBARA
}

\author{
Daryl Cooper \\ Department of Mathematics \\ University of California \\ Santa Barbara, CA 93106-3080 \\ cooper@math.ucsb.edu \\ Jiang-Hua Lu \\ Department of Mathematics \\ The University of Hong Kong \\ Pokfulam Rd., Hong Kong \\ jhlu@maths.hku.hk \\ Paul Yang \\ Department of Mathematics \\ Princeton University \\ Princeton NJ 08544-1000 \\ yang@math.princeton.edu
}

These supporting institutions contribute to the cost of publication of this Journal, but they are not owners or publishers and have no responsibility for its contents or policies.

See inside back cover or msp.org/pjm for submission instructions.

The subscription price for 2015 is US \$420/year for the electronic version, and \$570/year for print and electronic.

Subscriptions, requests for back issues and changes of subscribers address should be sent to Pacific Journal of Mathematics, P.O. Box 4163, Berkeley, CA 94704-0163, U.S.A. The Pacific Journal of Mathematics is indexed by Mathematical Reviews, Zentralblatt MATH, PASCAL CNRS Index, Referativnyi Zhurnal, Current Mathematical Publications and Web of Knowledge (Science Citation Index).

The Pacific Journal of Mathematics (ISSN 0030-8730) at the University of California, c/o Department of Mathematics, 798 Evans Hall \#3840, Berkeley, CA 94720-3840, is published twelve times a year. Periodical rate postage paid at Berkeley, CA 94704, and additional mailing offices. POSTMASTER: send address changes to Pacific Journal of Mathematics, P.O. Box 4163, Berkeley, CA 94704-0163.

PJM peer review and production are managed by EditFLOW ${ }^{\circledR}$ from Mathematical Sciences Publishers.

\section{PUBLISHED BY}

\section{mathematical sciences publishers \\ nonprofit scientific publishing}

http://msp.org/

(C) 2015 Mathematical Sciences Publishers 


\title{
PACIFIC JOURNAL OF MATHEMATICS
}

\author{
Volume 279 No. 1-2 December 2015
}

In memoriam: Robert Steinberg

Robert Steinberg (1922-2014): In memoriam V. S. VARADARAJAN

Cellularity of certain quantum endomorphism algebras

HENNING H. ANDERSEN, GUSTAV I. LEHRER and RUIBIN ZHANG

Lower bounds for essential dimensions in characteristic 2 via orthogonal representations ANTONIO BABIC and VLADIMIR CHERNOUSOV

Cocharacter-closure and spherical buildings

Michael Bate, Sebastian Herpel, Benjamin Martin and Gerhard RöHrLe

Embedding functor for classical groups and Brauer-Manin obstruction

Eva Bayer-Fluckiger, Ting-Yu LeE and Raman Parimala

On maximal tori of algebraic groups of type $G_{2}$

Constantin Beli, PhilipPe Gille and Ting-Yu LeE

On extensions of algebraic groups with finite quotient

MICHEL BRION

Essential dimension and error-correcting codes

SHANE CERNELE and ZiNOVy REICHSTEIN

Notes on the structure constants of Hecke algebras of induced representations of finite Chevalley groups

Charles W. CuRTis

Complements on disconnected reductive groups

FRANÇOIS DIGNE and JEAN MICHEL

Extending Hecke endomorphism algebras

Jie Du, Brian J. Parshall and LeOnard L. SCOTT

Products of partial normal subgroups

ELLEN HENKE

Lusztig induction and $\ell$-blocks of finite reductive groups

RADHA KESSAR and GUNTER MALLE

Free resolutions of some Schubert singularities

Manoj Kummini, Venkatramani Lakshmibai, Pramathanath Sastry and C. S. Seshadri

Free resolutions of some Schubert singularities in the Lagrangian Grassmannian

VenKatramani LAKSHMibai and ReUVEN HODGES

Distinguished unipotent elements and multiplicity-free subgroups of simple algebraic groups

Martin W. Liebeck, Gary M. Seitz and Donna M. Testerman

Action of longest element on a Hecke algebra cell module

GEORGE LUSZTIG

Generic stabilisers for actions of reductive groups

BENJAMIN MARTIN

On the equations defining affine algebraic groups

VLADIMIR L. POPOV

Smooth representations and Hecke modules in characteristic $p$

PETER SCHNEIDER

On CRDAHA and finite general linear and unitary groups

BHAMA SRINIVASAN

Weil representations of finite general linear groups and finite special linear groups PHAM HUU TIEP

The pro- $p$ Iwahori Hecke algebra of a reductive $p$-adic group, $\mathrm{V}$ (parabolic induction) MARIE-FRANCE VIGNÉRAS

Acknowledgement 